\title{
Huntington's Disease Clinical Trials Corner: January 2019
}

Filipe B. Rodrigues ${ }^{\mathrm{a}, \mathrm{b}, \mathrm{c}}$, Lori Quinn ${ }^{\mathrm{d}}$ and Edward J. Wild ${ }^{\mathrm{a}, *}$

${ }^{a}$ UCL Huntington's Disease Centre, UCL Queen Square Institute of Neurology, University College London, UK

${ }^{\mathrm{b}}$ Laboratory of Clinical Pharmacology and Therapeutics, Faculty of Medicine, University of Lisbon, PT

${ }^{\mathrm{c}}$ Clinical Pharmacology Unit, Instituto de Medicina Molecular, Lisbon, PT

${ }^{\mathrm{d}}$ Department of Biobehavioral Sciences, Teachers College, Columbia University, USA

\begin{abstract}
In this edition of the Huntington's Disease Clinical Trials Corner we expand on the GENERATION-HD1 and PACE-HD trials, and we list all currently registered and ongoing clinical trials in Huntington's disease.
\end{abstract}

Keywords: Huntington disease, clinical trials

\section{INTRODUCTION}

The Huntington's Disease Clinical Trials Corner is a regular section devoted to highlighting ongoing and recently completed clinical trials in Huntington's disease (HD). Clinical trials previously reviewed by the Huntington's Disease Clinical Trials Corner are listed in Table 1.

In this edition, we highlight the GENERATIONHD1 trial (NCT03761849) [1], and the PACE-HD trial (NCT03344601) [2], and briefly summarise the trial evidence around physiotherapy and exercise interventions in HD. Finally, we tabulate all currently registered and ongoing clinical trials in Tables 2 to 4. For further details on the methodology used, please refer to the first edition of Huntington's Disease Clinical Trials Corner [3].

If you would like to draw attention to specific trials, please feel free to email us at: f.rodrigues@ucl.ac.uk and e.wild@ucl.ac.uk.

In addition to the above, the published report of the PRIDE-HD trial (NCT02006472) is worthy of mention. The paper reports that "the study did not meet its primary of secondary endpoints at 26 weeks" [4],

\footnotetext{
*Correspondence to: Edward J. Wild, UCL Huntington's Disease Centre, 10-12 Russell Square, London, WC1B 5EH, UK. E-mail: e.wild@ucl.ac.uk.
}

confirming the results of previous trials [5-7] and suggests that pridopidine is unlikely have an effect on the motor symptoms of HD as assessed with the Unified Huntington's Disease Rating Scale (UHDRS) Total Motor Score (TMS).

\section{ONGOING CLINICAL TRIALS}

A list of all ongoing clinical trials is given in Tables 2-4.

\section{GENERATION-HDI (NCT03761849)}

Study title

A Randomized, Multicenter, Double-Blind, Placebo-Controlled, Phase III Clinical Study to Evaluate the Efficacy and Safety of Intrathecally Administered RO7234292 (RG6042) in Patients With Manifest Huntington's Disease [1].

\section{Intervention}

RG6042 (120 mg) - formerly known as IONIS$\mathrm{HTT}_{\mathrm{Rx}} /$ ISIS443139 - an antisense oligonucleotide that targets the HTT transcript allele-nonspecifically with the aim of lowering the production of mutant huntingtin protein [8]. 
Table 1

Clinical trials previously reviewed by the Huntington's Disease Clinical Trials Corner

\begin{tabular}{lccc}
\hline & Trial name & Intervention & Edition \\
\hline NCT02519036 & IONIS-HTTRx & IONIS-HTT $_{\text {Rx }} *$ & September 2017(3) \\
NCT02215616 & LEGATO-HD & Laquinimod & \\
NCT02197130 & Amaryllis & PF-02545920 & \\
NCT02006472 & PRIDE-HD & Pridopidine & \\
NCT03225833 & PRECISION-HD1 & WVE-120101 & February 2018(33) \\
NCT03225846 & PRECISION-HD2 & WVE-120102 & \\
NCT01795859 & FIRST-HD & Deutetrabenazine & \\
NCT02481674 & SIGNAL & VX15/2503 & August 2018(34) \\
NCT00712426 & CREST-E & Creatine & \\
NCT03761849 & GENERATION-HD1 & RG6042* & January 2018 \\
NCT03344601 & PACE-HD & Physical activity & \\
\hline
\end{tabular}

*IONIS-HTT Rx $_{\text {and }}$ R6042 refer to the same molecule.

\section{Description}

The GENERATION-HD1 trial, sponsored by Hoffmann-La Roche, aims to evaluate the efficacy, safety, and biomarker effects of monthly and bimonthly (i.e. every other month) $120 \mathrm{mg}$ of intrathecal RG6042 in adults (25 to 65 years of age) with manifest HD (i.e. a UHDRS Diagnostic Confidence Level of 4, a UHDRS Independence Score [IS] above or equal to 70, and a CAG-age Product equal or greater than 400) and intact functional independence at baseline to maintain self-care and core activities of daily living, comparing with intrathecal placebo, for disease modification.

This trial is a phase 3, international, multi-centre, randomized, placebo controlled, double-blind, parallel study. It will have 3 study arms: monthly intrathecal injections of $120 \mathrm{mg}$ RG6042; monthly intrathecal injections alternating between $120 \mathrm{mg}$ RG6042 and placebo; and monthly intrathecal placebo. The intervention will be administered for 25 months, and participants will be followed for 29 months. All participants are expected to be invited to an optional open-label extension (OLE) involving monthly or bimonthly (i.e. every other month) drug administration after the end of the blinded phase of the study, assuming the program is continuing.

The trial had not started recruitment at the time of writing, but has a recruitment target of 660 participants, over around 15 countries and 80 to 90 study sites, and it is planned to start enrolment by early 2019. It is currently public that recruitment will happen in the United States of America and Canada, where expected clinical sites were announced in December [9]. Details about further countries and sites will be released in the future.

This pivotal trial will have two primary clinical outcomes for regulatory purposes, the UHDRS Total Functional Capacity (TFC) for the FDA, and the composite UHDRS (cUHDRS) [10] for the EMA [11]. Secondary outcomes will involve other components of the UHDRS, clinical global impression, adverse events, the Montreal Cognitive Assessment (MoCA), the Columbia-Suicide Severity Rating Scale (C-SSRS), pharmacokinetic markers, cerebrospinal fluid mutant huntingtin and neurofilament light chain, and MRI brain volumes.

\section{Sponsors/funders: Hoffmann-La Roche}

\section{Comments}

This trial is the first to test huntingtin-lowering in a pivotal phase 3 trial, and is part of a development plan that includes the completed first-in-man phase $1 \mathrm{~b} / 2 \mathrm{a}$ IONIS-HTT $_{\text {RX }}$ (NCT02519036) trial [12], its ongoing OLE (NCT03342053) [13], the now-recruiting HD Natural History Study (NCT03664804) [14], and the imminent GENERATION-HD1 (NCT03761849) trial [1]. The phase 1b/2a involved 46 people with early stage HD and showed RG6042 to be safe and well-tolerated, and to reduce cerebrospinal fluid mutant huntingtin concentrations in a dose-dependent manner [15]. Results are currently being prepared for peer-reviewed publication [11]. After completion, all participants were invited to an OLE study aimed at studying long-term safety, tolerability, pharmacokinetics and pharmacodynamics of RG6042 over 15 months; this is currently ongoing. Participants entering the OLE were randomly allocated to monthly or bimonthly intrathecal doses of $120 \mathrm{mg}$ RG6042.

The HD Natural History Study is a prospective longitudinal observational study that aims to recruit 100 people with early stage $\mathrm{HD}$, matched individually to the participants of the open label extension study [16]. It aims to measure clinical and biomarkers (i.e. 


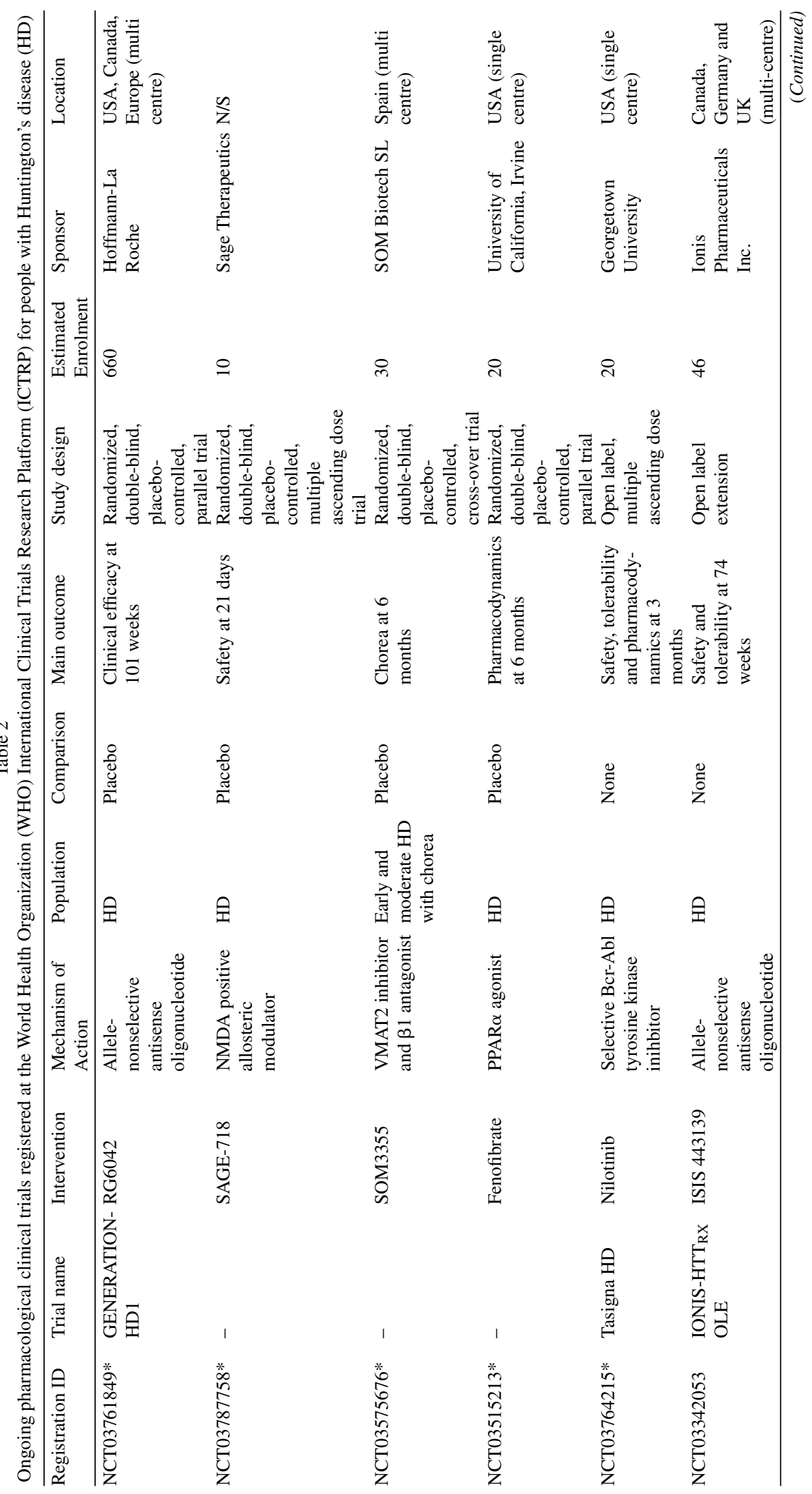




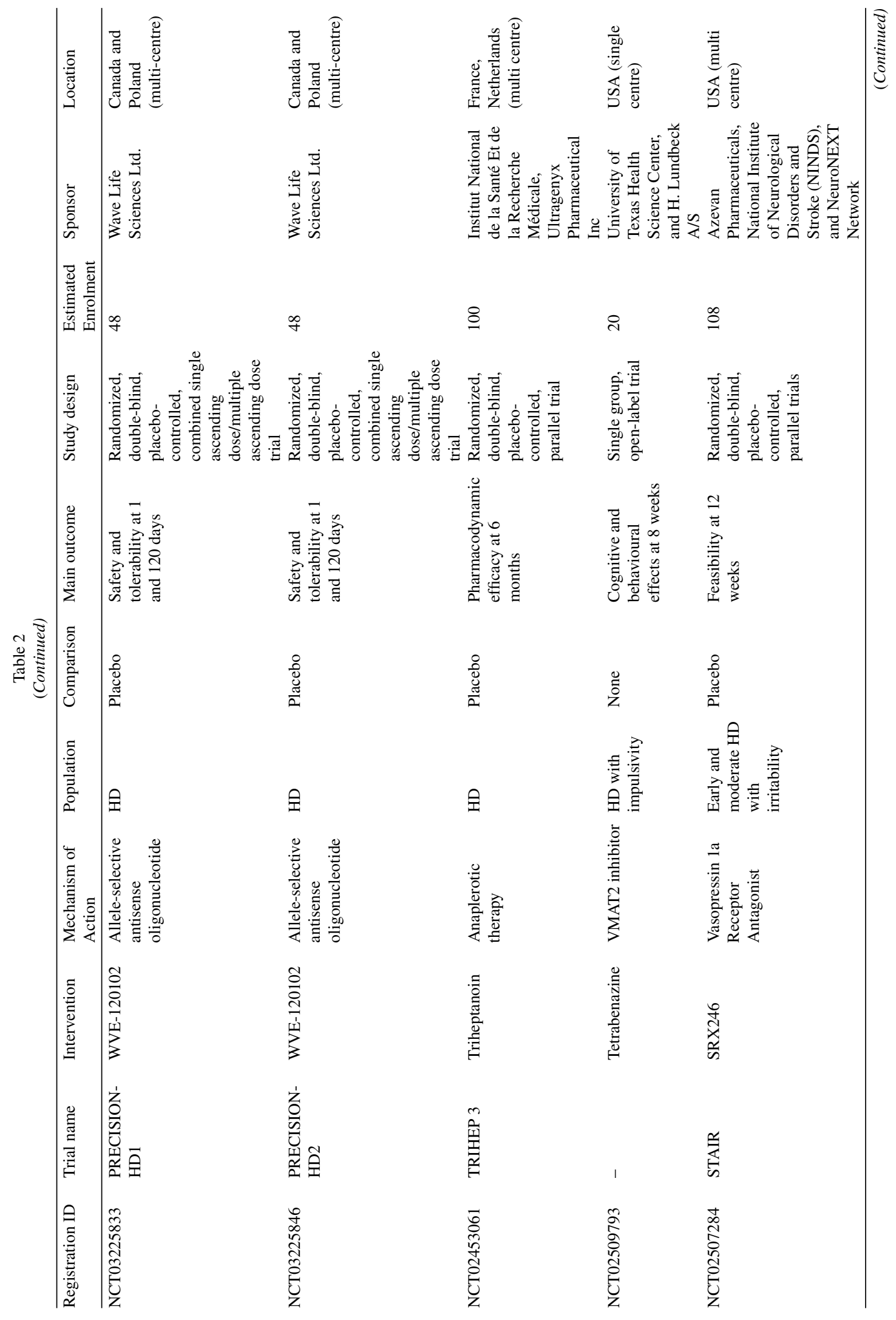


F.B. Rodrigues et al. / HD Clinical Trials Corner

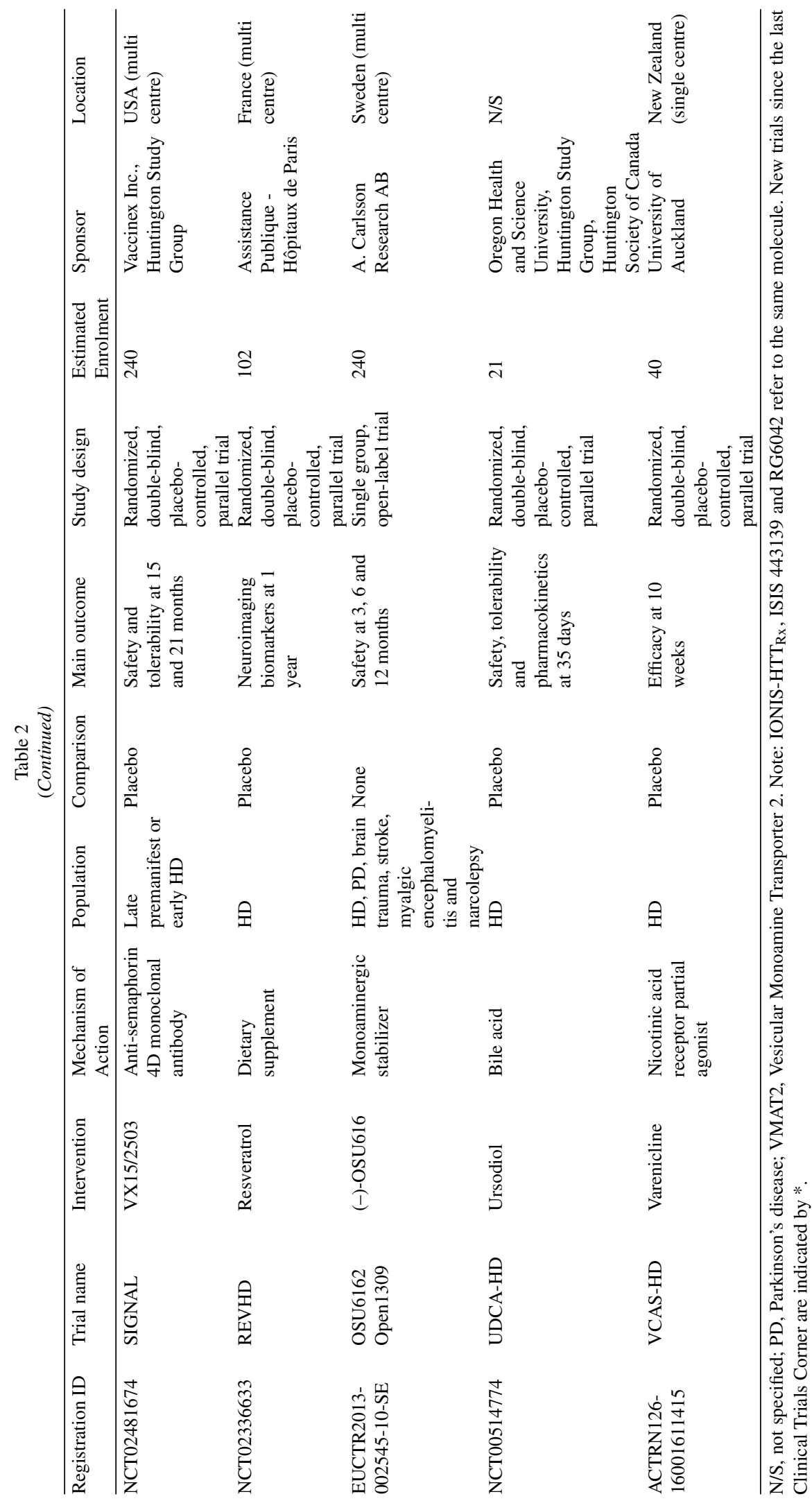




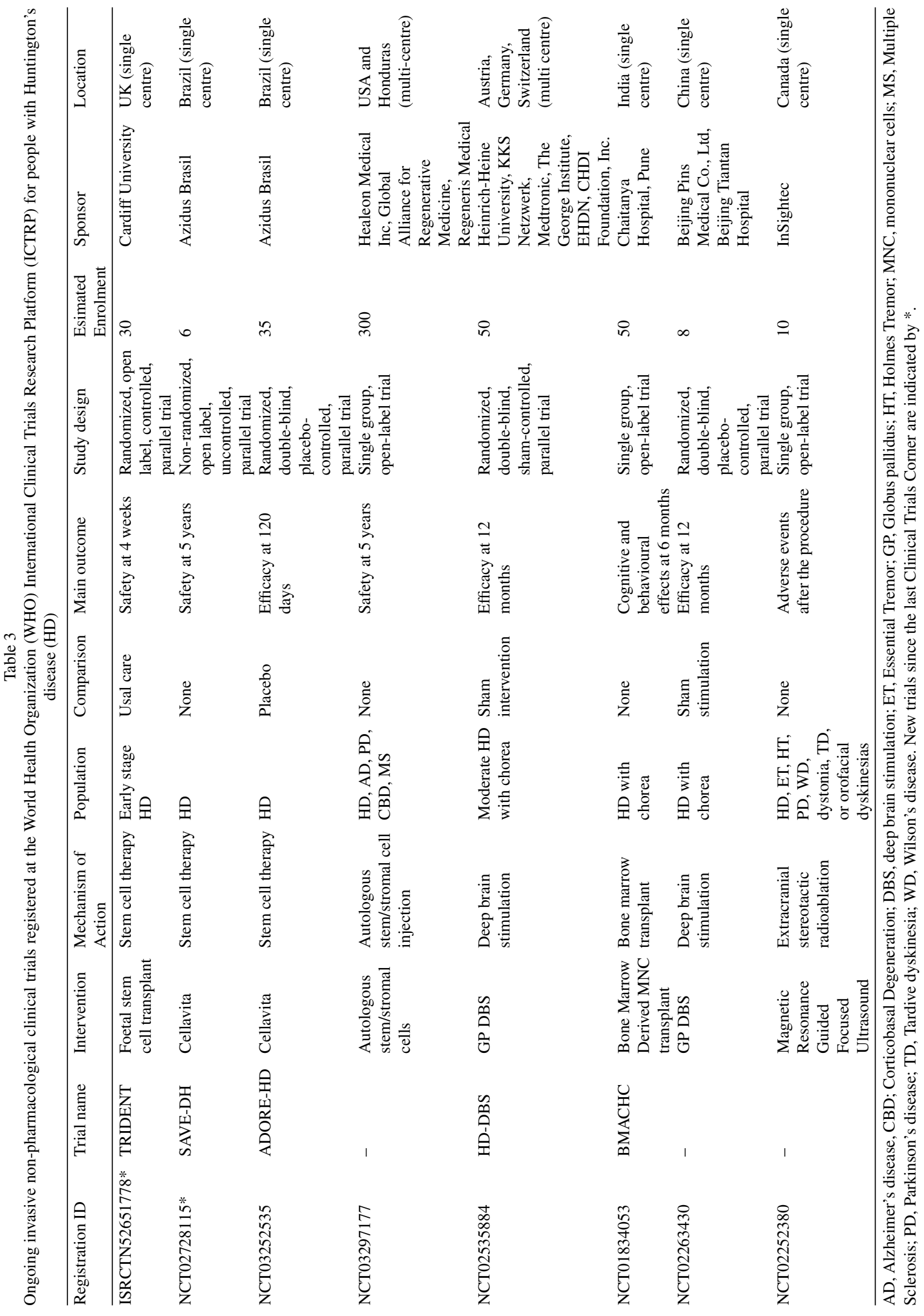




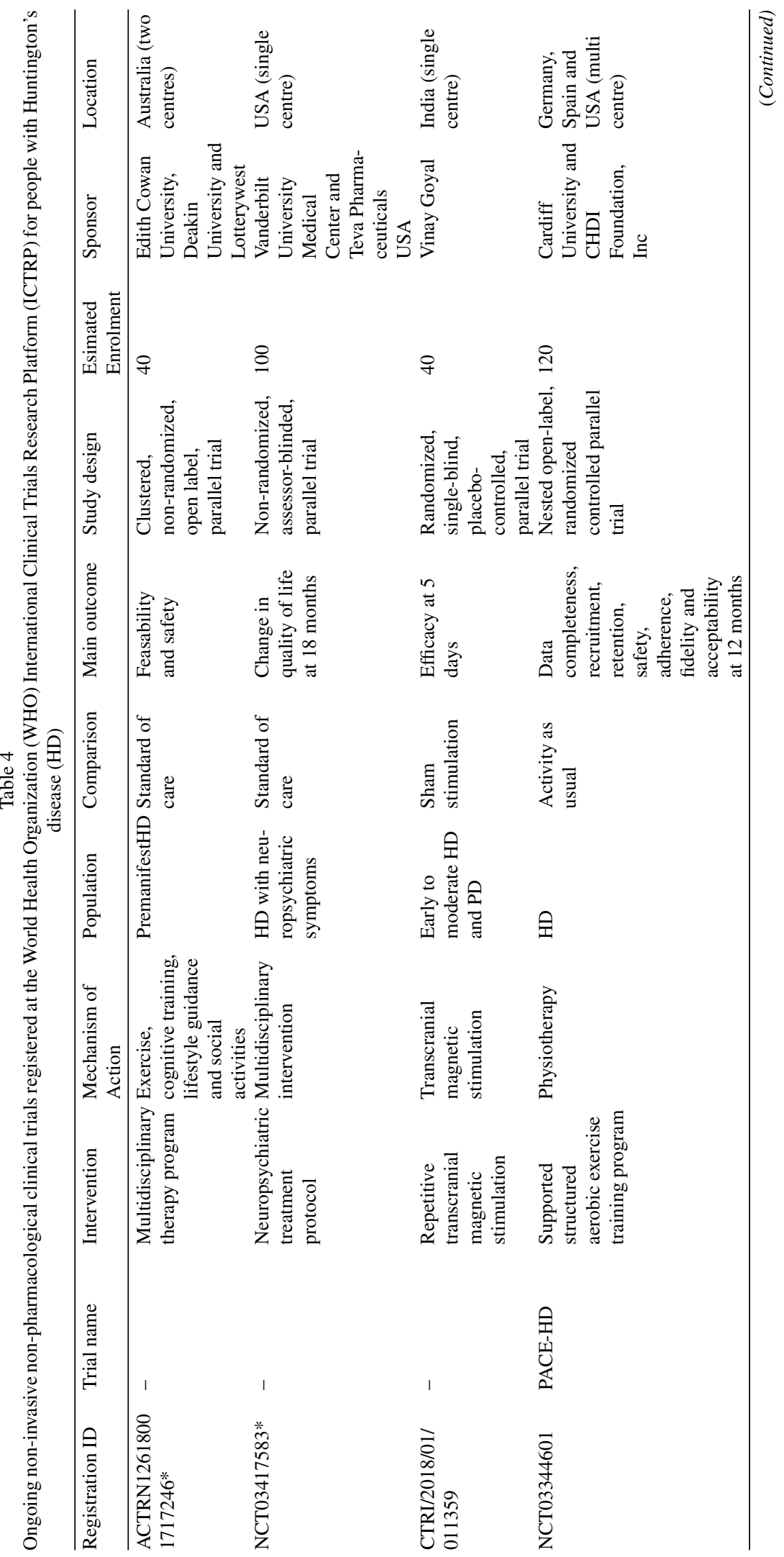




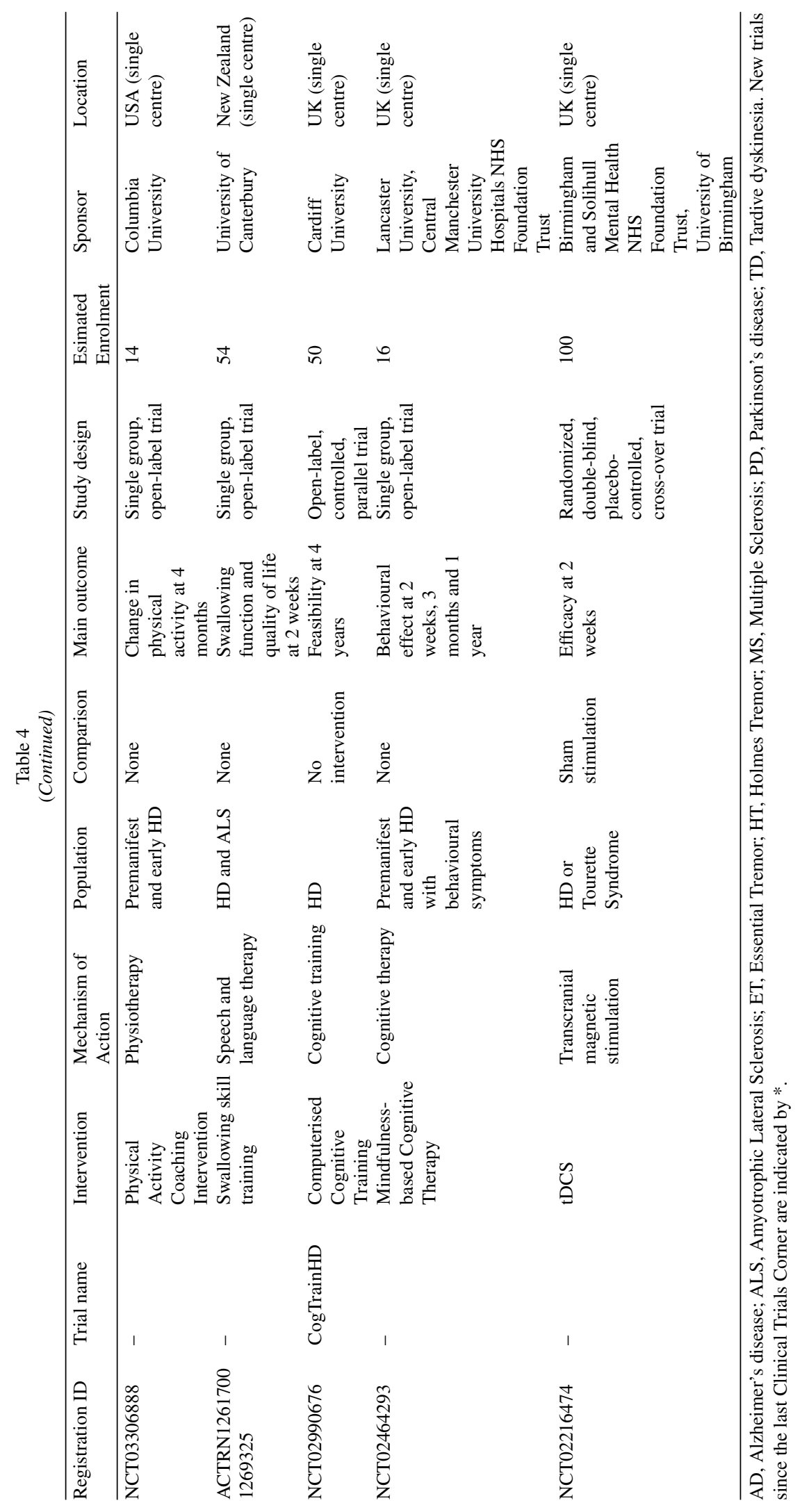


cerebrospinal fluid neurofilament light chain, mutant huntingtin and tau, brain MRI volumes, and digital biomarkers) over a 15-month period in a sample comparable to the phase $1 \mathrm{~b} / 2 \mathrm{a}$ and open label extension studies. Participants are being recruited in United States of America, Canada, Germany and United Kingdom. Participants will be offered continued open-label access to RG6042 after study termination.

Notably, for the pivotal phase 3 trial, GENERATION-HD1, Roche has opted for an enrichment strategy based on the inclusion criterion "CAG-age Product superior to 400". The CAG-age product (CAP score) is an estimate of lifetime exposure to mHTT toxicity [17], given by:

\section{[HTT CAG repeat length -33.66$] \times$ age}

Together with the remaining inclusion criteria, namely the UHDRS Diagnostic Confidence Level of 4, a UHDRS IS above or equal to 70, and functional independence at baseline, the use of a CAP score cutoff aims to produce a relatively homogeneous sample of early stage participants, whose expected progression during trial follow-up is greater and less variable [17]. This should improve the statistical power of the sample. It produces rigid minimum age cutoffs for each HTT CAG repeat length, as shown in Table 5.

Table 5

Minimum age at which individuals with each HTT CAG count will have a CAP score of $\geq 400$, permitting them to meet this inclusion criterion for Generation-HD1 and related trials

\begin{tabular}{lc}
\hline CAG & Minimum age \\
\hline 36 & Ineligible \\
37 & Ineligible \\
38 & Ineligible \\
39 & Ineligible \\
40 & 63.09 \\
41 & 54.50 \\
42 & 47.96 \\
43 & 42.83 \\
44 & 38.68 \\
45 & 35.27 \\
46 & 32.41 \\
47 & 29.99 \\
48 & 27.89 \\
49 & 26.08 \\
50 or over & 25 \\
\hline
\end{tabular}

Note that those with CAG counts of 36-39 will never meet this criterion while they still meet the maximum age inclusion criterion of 65 , while those with repeats of 50 or over all meet the CAP score cutoff, but would need to additionally meet the minimum age inclusion criterion of 25 .
This criterion will doubtless be a point of focus in discussions with potential volunteers.

\section{PACE-HD (NCT03344601)}

\section{Study title}

A Longitudinal Cohort Study With Nested Randomised Pragmatic Controlled Trial to Evaluate Physical Activity and Exercise Related Outcomes in People With Huntington's Disease (PACE-HD) [2].

\section{Intervention}

Supported structured aerobic exercise training program (18 face-to-face coaching sessions of $\sim 1$ hour).

\section{Description}

The PACE-HD trial, sponsored by Cardiff University and CHDI Foundation, Inc., aims to evaluate the feasibility, tolerability, and safety of supported structured aerobic exercise training program in adults $(\geq 18$ years of age) with genetically confirmed early manifest HD, compared with activity as usual.

PACE-HD is an international, multi-centre, observation study with a nested randomized, controlled, open label, parallel study. It will involve 120 participants, 60 of whom will take part on a longitudinal observational evaluation of physical fitness and physical activity over a period of 12 months, while the remaining 60 will be randomized to a supported structured aerobic exercise training program, or exercise as usual over a period of 12 months. Recruitment is currently open at various sites in the United States of America, Germany, and Spain.

The primary outcomes are data completeness, recruitment, retention, safety, adherence, fidelity, and acceptability. Secondary outcomes include exercise tests, walk endurance measures, the HD Pro-Triad, the Brunel Lifestyle Physical Activity Questionnaire, and digital biomarkers.

\section{Sponsors/funders: Cardiff University and CHDI} Foundation, Inc.

\section{Comments}

With multiple trials of agents intended to engage with the core pathobiology of HD underway, and more planned, the relevance of clinical interventions such as rehabilitation therapies, as both stand-alone and adjunctive therapies, has never been more significant. Similar to current management guidelines for Parkinson's disease [18] and multiple sclerosis [19], rehabilitation therapies - including physiotherapy, 
occupation therapy, exercise and physical activity - could be used alongside disease-modifying interventions with the potential to maximize patient outcomes.

Animal models of HD have provided pre-clinical evidence that exercise has the potential to modify disease progression. In R6/1 mice, sustained wheel running was shown to improve gait and motor coordination, as well as reduce striatal neuron loss [20]. More recent work with the longer life-span CAG140 mouse model demonstrated that 6 months of treadmill training resulted in increased striatal dopamine D2 receptor expression and dopamine neurotransmitter levels, reduction in HTT aggregate formation, as well as improved behavioural and cognitive symptoms [21]. These pre-clinical findings have set the stage for several clinical feasibility studies in people with early and moderate HD [22-25], as well as in several multi-disciplinary rehabilitation trials [26-30].

Combined pre-clinical and clinical data provide support for the evaluation of exercise as a therapeutic intervention strategy in HD. A recent systematic review reported on findings from 20 studies and found preliminary support for the benefits of exercise and physical activity in terms of motor function, gait speed, and balance [31, 32]. The review also reported a range of physical and social benefits identified through patient-reported outcomes. Interventions incorporating aerobic and strengthening exercises were most prevalent across studies, and several studies noted improvement or maintenance of motor function over 9 months or longer.

In order for rehabilitation interventions to be considered an important adjunctive therapy alongside pharmacological interventions, high-quality studies using innovative statistical methods and trials designs are needed. PACE-HD is a pragmatic study that includes both a longitudinal observational study and a nested (i.e. within-cohort) randomized controlled trial of a 12-month physical therapy and exercise intervention. The intervention incorporates the use of wearable physical activity monitors to measure both outcomes and activity levels throughout the trial. The study is conducted alongside Enroll-HD, which minimizes subject burden and will provide a basis for comparative analysis on disease progression measures. Results of this study are due in the summer of 2020.

A formal Clinical Guideline for Exercise in HD is currently in development. This will provide evidencebased recommendations for healthcare providers and persons with $\mathrm{HD}$, and is planned to be available later in 2019.

\section{ACKNOWLEDGMENTS}

The authors are supported by CHDI Foundation, Inc. (salary support to FBR for conduct of the HDClarity study), Medical Research Council UK (salary support to EJW) and the Jacques and Gloria Gossweiler Foundation (salary support to LQ).

\section{CONFLICTS OF INTEREST}

FBR and EJW were sub-investigators on LEGATO-HD (NCT02215616) and IONIS HTT Rx (NCT02519036), and are sub-investigators on the

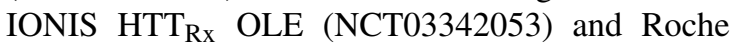
Natural History Study (NCT03664804) trials, and EJW was a sub-investigator on the Amaryllis study (NCT02197130). The authors did not make use of confidential or privileged information: all materials included in this manuscript were collected from publicly available sources. EJW has participated in scientific advisory boards with Hoffmann-La Roche Ltd, Ionis, Shire, GSK, Wave Life Sciences, PTC Therapeutics and Mitoconix. All honoraria were paid through UCL Consultants Ltd, a wholly owned subsidiary of UCL. Their Host Institution, University College London Hospitals NHS Foundation Trust, has received funds as compensation for conducting clinical trials for Ionis Pharmaceuticals, Pfizer and Teva Pharmaceuticals. Hoffman La Roche Ltd has supported UCL with research funding for EJW. LQ received honoraria from the Huntington Study Group and royalties from Elsevier Publishing.

\section{REFERENCES}

[1] Hoffmann-La Roche. A Study to Evaluate the Efficacy and Safety of Intrathecally Administered RO7234292 (RG6042) in Patients With Manifest Huntington's Disease. https://ClinicalTrials.gov/show/NCT03761849; 2018.

[2] Cardiff University, CHDI Foundation Inc. PHysical Activity and Exercise Outcomes in Huntington's Disease. https://ClinicalTrials.gov/show/NCT03344601; 2018.

[3] Rodrigues FB, Wild EJ. Clinical Trials Corner: September 2017. Journal of Huntingtons Disease. 2017;6(3):255-63.

[4] Reilmann R, McGarry A, Grachev ID, Savola J-M, Borowsky B, Eyal E, et al. Safety and efficacy of pridopidine in patients with Huntington's disease (PRIDE-HD): A phase 2 , randomised, placebo-controlled, multicentre, doseranging study. Lancet Neurology. 2018.

[5] de Yebenes JG, Landwehrmeyer B, Squitieri F, Reilmann $\mathrm{R}$, Rosser A, Barker RA, et al. Pridopidine for the treatment of motor function in patients with Huntington's disease (MermaiHD): A phase 3, randomised, double-blind, placebo-controlled trial. Lancet Neurology. 2011;10(12): 1049-57. 
[6] Huntington Study Group HART Investigators. A randomized, double-blind, placebo-controlled trial of pridopidine in Huntington's disease. Movement Disorders. 2013;28(10): 1407-15.

[7] Lundin A, Dietrichs E, Haghighi S, Goller ML, Heiberg A, Loutfi G, et al. Efficacy and safety of the dopaminergic stabilizer Pridopidine (ACR16) in patients with Huntington's disease. Clinical Neuropharmacol. 2010;33(5):260-4.

[8] Wild EJ, Tabrizi SJ. Therapies targeting DNA and RNA in Huntington's disease. Lancet Neurology. 2017;16(10):83747.

[9] HDSA. RG6042 GENERATION HD1 Study: Expected Sites in USA \& Canada 2018 [Available from: https://hdsa. org/wp-content/uploads/2018/12/RG6042-

GENERATION-HD1-Study-update-12.19.2018.pdf.

[10] Schobel SA, Palermo G, Auinger P, Long JD, Ma S, Khwaja OS, et al. Motor, cognitive, and functional declines contribute to a single progressive factor in early HD. Neurology. 2017;89(24):2495-502.

[11] Schobel S, Palermo G, Trundell D, Kremer T, SanwaldDucray P, Smith A, et al., editors. A Global Development Program Testing RG6042, an Antisense Oligonucleotide, for the Treatment of Early Manifest Huntington's Disease. European Huntington's Disease Network 2018 Plenary Meeting; 2018 14-16 September 2018; Vienna, Austria.

[12] Ionis Pharmaceuticals I. Safety, Tolerability, Pharmacokinetics, and Pharmacodynamics of IONIS-HTTRx in Patients With Early Manifest Huntington's Disease. https://ClinicalTrials.gov/show/NCT02519036; 2015.

[13] Ionis Pharmaceuticals I. Study in Huntington's Disease Patients Who Participated in Prior Investigational Studies of ISIS 443139. https://ClinicalTrials.gov/show/ NCT03342053; 2017.

[14] Hoffmann-La Roche. Study to Measure Cerebrospinal Fluid Mutant Huntingtin Protein in Participants With Early Manifest Stage I or Stage II Huntington's Disease. https://ClinicalTrials.gov/show/NCT03664804; 2018.

[15] Tabrizi S, Leavitt B, Kordasiewicz H, Czech C, Swayze E, Norris DA, et al. Effects of IONIS-HTTRx in patients with early huntington's disease, results of the first HTT-lowering drug trial. Neurology. 2018;90.

[16] Hooper G, Trundell D, Palermo G, Kremer T, Frick E, Boak L, et al. Design of a prospective, longitudinal, natural history study in huntington disease. Neurotherapeutics. 2018;15(4):1182-3.

[17] Long JD, Landwehrmeyer B, Palermo G, Schobel S, Tabrizi SJ. Enrichment strategy in early-to-moderate manifest huntington disease based on CAG/Age product (CAP) $>400$ threshold. Neurotherapeutics. 2018;15(4):1186.

[18] National Institute for Health and Care Excellence. Parkinson's disease in adults (NG71) 2017 [Available from: nice.org.uk/guidance/ng71.

[19] National Institute for Health and Care Excellence. Multiple sclerosis in adults: Management (CG186) 2014 [Available from: nice.org.uk/guidance/cg186.

[20] Harrison DJ, Busse M, Openshaw R, Rosser AE, Dunnett $\mathrm{SB}$, Brooks SP. Exercise attenuates neuropathology and has greater benefit on cognitive than motor deficits in the R6/1 Huntington's disease mouse model. Experimental Neurology. 2013;248:457-69.

[21] Stefanko DP, Shah VD, Yamasaki WK, Petzinger GM, Jakowec MW. Treadmill exercise delays the onset of nonmotor behaviors and striatal pathology in the CAG140 knock-in mouse model of Huntington's disease. Neurobiology of Disease. 2017;105:15-32.
[22] Busse M, Quinn L, Debono K, Jones K, Collett J, Playle $\mathrm{R}$, et al. A randomized feasibility study of a 12-week community-based exercise program for people with Huntington's disease. Journal of Neurologic Physical Therapy. 2013;37(4):149-58.

[23] Quinn L, Debono K, Dawes H, Rosser AE, Nemeth AH, Rickards H, et al. Task-specific training in Huntington disease: A randomized controlled feasibility trial. Physical Therapy. 2014;94(11):1555-68.

[24] Quinn L, Hamana K, Kelson M, Dawes H, Collett J, Townson J, et al. A randomized, controlled trial of a multi-modal exercise intervention in Huntington's disease. Parkinsonism \& Related Disorders. 2016;31:46-52.

[25] Busse M, Quinn L, Drew C, Kelson M, Trubey R, McEwan $\mathrm{K}$, et al. Physical activity self-management and coaching compared to social interaction in huntington disease: Results from the ENGAGE-HD randomized, controlled pilot feasibility trial. Physical Therapy. 2017;97(6): 625-39.

[26] Thompson JA, Cruickshank TM, Penailillo LE, Lee JW, Newton RU, Barker RA, et al. The effects of multidisciplinary rehabilitation in patients with early-to-middle-stage Huntington's disease: A pilot study. European Journal of Neurology. 2013;20(9):1325-9.

[27] Piira A, van Walsem MR, Mikalsen G, Nilsen KH, Knutsen $\mathrm{S}$, Frich JC. Effects of a one year intensive multidisciplinary rehabilitation program for patients with huntington's disease: A prospective intervention study. PLoS Currents. 2013;5.

[28] Piira A, van Walsem MR, Mikalsen G, Oie L, Frich JC, Knutsen S. Effects of a two-year intensive multidisciplinary rehabilitation program for patients with huntington's sisease: A prospective intervention study. PLoS Currents. 2014;6.

[29] Ciancarelli I, De Amicis D, Di Massimo C, Sandrini G, Pistarini C, Carolei A, et al. Influence of intensive multifunctional neurorehabilitation on neuronal oxidative damage in patients with Huntington's disease. Functional Neurology. 2015;30(1):47-52.

[30] Cruickshank TM, Thompson JA, Dominguez DJ, Reyes AP, Bynevelt M, Georgiou-Karistianis N, et al. The effect of multidisciplinary rehabilitation on brain structure and cognition in Huntington's disease: An exploratory study. Brain and Behavior. 2015;5(2):e00312.

[31] Fritz NE, Rao AK, Kegelmeyer D, Kloos A, Busse M, Hartel L, et al. Physical therapy and exercise interventions in huntington's disease: A mixed methods systematic review. Journal of Huntingtons Disease. 2017;6(3):217-35.

[32] Quinn L, Busse M, Carrier J, Fritz N, Harden J, Hartel L, et al. Physical therapy and exercise interventions in Huntington's disease: A mixed methods systematic review protocol. JBI Database of Systematic Reviews and Implementation Reports. 2017;15(7):1783-99.

[33] Rodrigues FB, Wild EJ. Huntington's Disease Clinical Trials Corner: February 2018. Journal of Huntingtons Disease. 2018;7(1):89-98.

[34] Rodrigues FB, Wild EJ. Huntington's Disease Clinical Trials Corner: August 2018. Journal of Huntingtons Disease. 2018;7(3):279-86. 